\title{
Smoking and physical inactivity increase cancer prevalence in BRCA-1 and BRCA-2 mutation carriers: results from a retrospective observational analysis
}

\author{
Sabine Grill ${ }^{1} \quad$ Maryam Yahiaoui-Doktor $^{2} \cdot$ Ricarda Dukatz $^{1} \cdot$ Jacqueline Lammert $^{1} \cdot$ Mirjam Ullrich $^{1}$. \\ Christoph Engel $^{2} \cdot$ Katharina Pfeifer $^{1} \cdot$ Maryam Basrai $^{3} \cdot$ Michael Siniatchkin $^{4} \cdot$ Thorsten Schmidt $^{5}$. \\ Burkhard Weisser $^{5} \cdot$ Kerstin Rhiem $^{6} \cdot$ Nina Ditsch $^{7} \cdot$ Rita Schmutzler $^{6} \cdot$ Stephan C. Bischoff $^{3} \cdot$ Martin Halle $^{8}$. \\ Marion Kiechle ${ }^{1}$
}

\begin{abstract}
Background The aim of this analysis in a pilot study population was to investigate whether we can verify seemingly harmful lifestyle factors such as nicotine and alcohol indulgence, obesity, and physical inactivity, as well as a low socioeconomic status for increased cancer prevalence in a cohort of BRCA 1 and 2 mutation carriers.

Methods The analysis data are derived from 68 participants of the lifestyle intervention study LIBRE-1, a randomized, prospective trial that aimed to test the feasibility of a lifestyle modification in BRCA 1 and 2 mutation carriers. At study entry, factors such as medical history, lifestyle behavior, and socioeconomic status were retrospectively documented by interview and the current BMI was determined by clinical examination. The baseline measurements were compared within the cohort, and presented alongside reference values for the German population.

Results Study participants indicating a higher physical activity during their adolescence showed a significantly
\end{abstract}

Sabine Grill

Sabine.Grill@1rz.tum.de

1 Department of Gynecology and Center for Hereditary Breast and Ovarian Cancer, Klinikum Rechts der Isar, Technical University of Munich (TUM) and Comprehensive Cancer Center Munich (CCCM), Ismaninger Str. 22, 81675 Munich, Germany

2 Institute for Medical Informatics, Statistics and Epidemiology, University of Leipzig, Haertelstrasse 16-18, 04107 Leipzig, Germany

3 Institute of Nutritional Medicine, University of Hohenheim, Fruwirthstr. 12, 70593 Stuttgart, Germany

4 Institute for Medical Psychology and Sociology, University Hospital Schleswig-Holstein, Campus Kiel, Preusserstr. 1-9, 24105 Kiel, Germany lower cancer prevalence $(p=0.019)$. A significant difference in cancer occurrence was observed in those who smoked prior to the disease, and those who did not smoke $(p<0.001)$. Diseased mutation carriers tended to have a lower BMI compared to non-diseased mutation carriers ( $p=0.079$ ), whereas non-diseased revealed a significantly higher physical activity level than diseased mutation carriers $(p=0.046)$.

Discussion The present data in this small cohort of 68 mutation carriers suggest that smoking and low physical activity during adolescence are risk factors for developing breast cancer in women with BRCA1 or BRCA2 mutation. Further data of the ongoing LIBRE 2 study are necessary to confirm these findings in a larger cohort of 600 mutation carriers.

Keywords BRCA1 - BRCA2 - Physical activity · Lifestyle $\cdot$ Smoking $\cdot$ Cancer prevalence

5 Department of Sports Medicine, Christian-Albrecht University Kiel, Olshausenstraße 74, 24098 Kiel, Germany

6 Center for Hereditary Breast and Ovarian Cancer, University Hospital Cologne, Kerpener Str. 34, 50931 Cologne, Germany

7 Department for Obstetrics and Gynecology, University Hospital Munich-Grosshadern, Ludwig-Maximilians-Univers ity, Marchioninistraße 15, 81377 Munich, Germany

8 Department of Prevention and Sports Medicine, Klinikum Rechts der Isar, Technical University of Munich (TUM), Ismaninger Str. 22, 81675 Munich, Germany 


\section{Background}

Breast cancer is the most common cancer among women especially in developed countries, with an estimated 1.7 million new cases diagnosed worldwide each year [1]. Incidence rates vary nearly fourfold among different regions of the world [1], which obviously reflects differences in reproductive and lifestyle risk factors such as obesity, inactivity, and hypercaloric nutrition [2]. Therefore, these factors warrant special scientific interest. Numerous prospective studies have demonstrated that physical activity, the dietary pattern, and body weight, but also the social environments have a significant impact on the incidence of sporadic breast cancer, coping, and prognosis [2]. To what extent lifestyle factors can even intervene in the well-being of patients with genetic predisposition such as BRCA1 or BRCA 2 mutation has hardly been studied.

Familial susceptibility to breast cancer accounts for approximately $25 \%$ of all breast cancer cases, $30 \%$ of which is attributed to the cancer susceptibility genes BRCA1 and BRCA2 [3]. These germline mutations are transmitted as an autosomal dominant form with incomplete penetrance. BRCA1 and BRCA2 are tumor suppressor genes that encode proteins that act in common pathways of genome protection and play important roles at different stages in DNA damage response and DNA repair [3]. Mutations in these genes confer a high risk of breast as well as ovarian cancer. The average cumulative cancer risk by the age of 70 has been estimated to be 80 and $55 \%$, respectively, for BRCA1 mutation carriers and 60 and $16.5 \%$, respectively, for BRCA2 mutation carriers [4]. Ovarian carcinoma occurs at a younger age in BRCA1 mutation carriers than in BRCA2 mutation carriers or the general population [5].

Current literature implies that the risk of developing cancer in gene carriers may be influenced through genetic factors (polymorphisms), as well as exogenous factors such as reproductive factors, lifestyle, and physical activity during adolescence [6-9]. The lifetime cancer risk ranges from 30 to $80 \%$ [6-9]. It also raises the question whether disease progression is influenced by the socioeconomic background. Lifestyle modulating factors such as smoking, alcohol consumption, eating habits, and physical activity are unevenly distributed among socioeconomic classes, and an influence on breast cancer risk is suspected [10-12].

So far, there are only few prospective studies examining the influence of the socioeconomic background, as well as modulating risk factors in women with BRCA 1 and 2 mutations. Overall, the importance of hereditary breast and ovarian cancer has been growing, since recent studies assume an increasing penetrance of inherited BRCA1 mutations as generations proceed $[6,13,14]$. Antoniou et al. postulate an increase of breast cancer penetrance at the range of 24-67\% for female carrier of a BRCAl mutation, born after 1940 .
Similar results were observed in an Icelandic cohort. The penetrance of the mutation has quadrupled in female $B R C A 1$ mutation carriers born before versus after 1920, namely $18.6 \%$ (95\% CI $11.0-29.5 \%$ ) up to a value of $71.9 \%$ (95\% CI $45.9-100 \%)(p<0.001)$ [15]. A possible explanation for the increased penetrance may be a change of reproductive behavior [16], lifestyle, and social environment over the years.

The scientific analysis of the social composition, as well as the detection of a possible interaction of personal risk factors, lifestyle, and environmental determinants has achieved a unique degree of importance to prevent mutation carriers from developing cancer. Focusing on health consciousness and socioeconomic background, we aimed to analyze these factors in our study on a BRCA1 and 2 positive cohorts [17] and accentuate the differences to the age-adapted female German population.

\section{Subjects and methods}

\section{Study cohort and patient recruitment}

The BRCA 1 and BRCA 2 female mutation carriers described in this analysis were participants of the lifestyle intervention study LIBRE-1. A multicentre, interdisciplinary, prospective, open study design was used with two arms, randomizing participants into a control and an intervention group with a ratio of 1:1. LIBRE-1 aimed to analyze the feasibility of a lifestyle intervention for non-diseased and diseased BRCA mutation carriers in the form of a supervised physical training program and nutrition education focusing on the Mediterranean dietary pattern [17]. A large consecutive efficacy study including 600 mutation carriers is planned in the scope of the LIBRE 2 study [18].

The study was conducted at three study centers of the German Consortium of Hereditary Breast and Ovarian Cancer (GC-HBOC, http://www.konsortium-familiaererbrustkrebs.de), Cologne, Kiel, and Munich. From February to July 2014, 68 patients with a pathogenic BRCA gene mutation were recruited for the clinical study. Inclusion criteria were a documented pathogenic BRCA 1 or BRCA 2 germline mutation, age over 18 , and written informed consent. Exclusion criteria were an above-average physical fitness at the baseline visit with a ventilatory threshold VT1 in spiroergometry $\left(V \mathrm{O}_{2 \max }\right)>150 \%$ of the general population at the baseline visit and different factors that interfere with a successful intervention: metastatic tumor disease, life expectancy $<3$ years, limiting cardiovascular and lung diseases (instable coronary heart disease, heart failure NYHA stage IV, chronic obstructive pulmonary disease (COPD) GOLD IV, maximal resting blood pressure at rest $>160 / 100 \mathrm{mmHg}$ ), significant orthopaedic 
problems, serious diseases, not allowing a participation in group interventions (e.g., psychiatric or internal ailment), a Carnovsky-Index $<60 \%$ [19], an exercise capacity $<50 \mathrm{~W}$, food allergies not allowing consumption of a Mediterranean dietary pattern, a vegan diet, BMI $<15 \mathrm{~kg} / \mathrm{m}^{2}$, current pregnancy, insufficient knowledge of the German language, unwillingness to cooperate, and current participation in other lifestyle intervention trials.

The patients were recruited from the GC-HBOC register, from interdisciplinary genetic counseling units at the hospitals and via advertising campaigns in print media, on the Internet and at patient information events. Eligible patients received an information package, consisting of patient information, the written consent form, and the study flyer.

\section{Approval, registration, and funding}

The local Ethical Committees of the Universities of Munich, Kiel, and Cologne approved the study protocol [17], and written informed consent was obtained from all participants before entry into the study. The study has been registered at the German study register for clinical studies DRKS https://drks-neu.uniklinik-freiburg.de/ drks_web/navigate.do?navigationId=trial.HTML\&TRIAL_ ID=DRKS00005736), at the study register of the National Institutes of Health (clinicaltrials.gov Identifier: NCT02087592) and at the WHO (http://apps.who.int/trialsearch/Trial.aspx?TrialID $=$ NCT02087592) and has been funded by the German Cancer Aid as a focus project for primary prevention (funding code 110013).

Within this clinical analysis, the impact of lifestyle risk factors and socioeconomic status was evaluated for our study cohort of non-cancer and cancer diseased BRCA mutation carriers, and a comparison was made to the age-adapted female German reference group according to the recent publication of the German Federal Statistical Office.

\section{Assessment of the clinical baseline data}

We conducted a baseline survey at the first study visit. Two interview forms were used to record the patient's medical history, disease status, and risk factors for carcinogenesis such as hormone replacement, reproduction, and behavioral factors of her lifestyle (clinical baseline questionnaire "KBAS"). Furthermore, the familial and social background, the grade of education, and the participant's income were encompassed (sociodemographic questionnaire "SOZ").

To record the smoking behavior, we asked the participating mutation carriers if they had ever smoked (yes/no), age of smoking begin, and the quantity and duration of the cigarette consumption, and if they were currently smoking. In addition, the participants were interviewed about their activity patterns in everyday life. Therefore, we assessed the time which they spent with bike riding, walking, gardening, fitness, and housework in winter and summer months on a daily base. Moreover, within the baseline questionnaire, study participants were requested to compare their individual activity pattern to age peers (clearly less active/slightly less active/the same level of activity/clearly more active than other women at the age of 10-19, 20-29, 30-39, 40-49, 50-59, and 60-69). Furthermore, alcohol consumption habits were investigated including the participants' age when she started to consume alcoholic beverages, the quantity, and alcoholic content that is consumed on a regular base (glasses of wine, aperitif, beer, and spirituous beverages/rarely or never/ 1 per month to $<1$ per week $/ 1-5$ per week/6-10 per week/10-15 per week/> 15 per week). These data were converted into grams ethanol per day ( $\mathrm{g} /$ day).

Besides that the mutation carriers were interviewed about their graduation degree and their income, divided into own salary and household income, to assess their socioeconomic level.

\section{Statistical analysis}

We used the electronic study management software "OpenClinica" for the documentation of study visits. The statistical analysis was carried out after the database finalization at 07/01/2016. The statistical analysis was carried out using IBM SPSS 23. The planned number of patients for this feasibility analysis was 60 . As the study was well accepted and the demand for participation was high, the recruited number of patients was 68 .

Our analysis was of a descriptive nature, comparing the two groups diseased and healthy mutation carriers with one another. Where the parameters being analyzed were binary items, we applied a Chi-square test, and for metric parameters, we applied a $t$ test for independent samples. As we view this work as hypothesis generating for the larger LIBRE 2 study, we decided not to apply a correction on the level of statistical significance, which was 0.05 .

\section{Results}

Clinical data records were available for all subjects $(n=68)$ at study enrollment. Of the 68 participants, 8 were enrolled in Cologne, 23 in Kiel, and 37 in Munich. Thirty five of the participating women were randomized to the control group, and 33 to the intervention group.

\section{Baseline data}

Clinical data: age, prophylactic surgery, mutation, and cancer status

At study entry, the baseline median age of the study subjects was 41.5 years ranging from 24 to $72.61 .8 \%(n=42)$ of the 
patients showed a mutation in the BRCA1 gene, whereas $38.2 \%(n=26)$ were carriers of a BRCA 2 mutation. Fortysix $(67.6 \%)$ participants had previously developed cancer including three cases of ovarian cancer and 43 cases of breast cancer. As far as surgical interventions are concerned, 31 women (45.6\%) had already undergone an adnexectomy mostly in terms of prophylactic bilateral salpingo-oophorectomy (PBSO), whereas 21 women (30.9\%) had received a mastectomy, among them 7 cases with unilateral and 14 cases with bilateral mastectomy.

Lifestyle factors: smoking, BMI, physical activity, and alcohol consumption

At the time of study enrollment, eight participants were current smokers, 24 non-smokers, and 36 were former smokers. Sixty seven indicated intermittent alcohol consumption, of mostly wine or beer. Considering spirituous beverages, excessive drinking behavior was not reported.

Emphasizing physical constitution, clinical examination revealed a median BMI of $23.2 \mathrm{~kg} / \mathrm{m}^{2}$ determined within a range of 18 and $45 \mathrm{~kg} / \mathrm{m}^{2}$ in our study population of mutation carriers.

With reference to the statements about physical activity, $51 \%$ of the participating mutation carriers spent $1-3 \mathrm{~h}$ on physical training per week, whereas $21 \%$ showed an even higher level of activity consisting of 3-5 h/week. Ten percent of the mutation carriers stated a maximum of more than $5 \mathrm{~h}$ of physical training per week. Sixteen percent admitted to not doing any sports, and $2 \%$ did not provide information about their physical activity.

Evaluating the activity pattern in childhood and adolescence, defined as the time period between the age of 10-19, in our study, $55.1 \%$ of the mutation carriers stated to have participated in more than $3 \mathrm{~h}$ of sports per week, in contrast to $13 \%$ that admitted a lower level of physical activity during childhood and adolescence consisting of $2 \mathrm{~h}$ and $1 \mathrm{~h} /$ week, respectively, for another $13 \%$. However, another $18.8 \%$ performed less than $1 \mathrm{~h}$ of physical exercise per week.

\section{Socioeconomic factors: income and education}

At study entry, 42 out of 68 provided information about their socioeconomic background. The mean net income was stated to be $€ 3700$ at the time of study enrollment.

Referring to educational level, $47 \%$ of the mutation carriers had a very high level of education, $13 \%$ a high level, and $40 \%$ indicated to have a low level of education.

\section{Baseline data compared to the German population (Table 1)}

\section{$B M I$}

Clinical examination revealed a median BMI of $23.2 \mathrm{~kg} / \mathrm{m}^{2}$ in our study population of mutation carriers. In this regard, the mean BMI in this study was well below the average of the female German peer group according to the latest release of the German Federal Ministry of Health 2013, which is specified in $25.7 \mathrm{~kg} / \mathrm{m}^{2}$ comprising women at the age of $41 \pm 10$ years $\left(24.9 \mathrm{~kg} / \mathrm{m}^{2}\right.$ in women at the age of $30-35$, $25.4 \mathrm{~kg} / \mathrm{m}^{2}$ in women at the age $35-40,25.7 \mathrm{~kg} / \mathrm{m}^{2}$ in women at the age $40-45,25.9 \mathrm{~kg} / \mathrm{m}^{2}$ in women at the age $45-50$, and BMI $26.7 \mathrm{~kg} / \mathrm{m}^{2}$ in women at the age 55-60).

\section{Physical activity}

Comparing our results of physical activity to the average German activity level, clear differences could be noticed as well. According to the Robert Koch Institute 2013, in

Table 1 Comparison of the study group with the German population

\begin{tabular}{|c|c|c|c|c|}
\hline Parameter & LIBRE study group & Reference value & Cohort & Source \\
\hline Physical activity (h/week) & & & 40-49-year-old German females & Robert Koch Institute 2013 \\
\hline$<2.5$ & $51 \%$ & $83 \%$ & & \\
\hline$>2.5$ & $49 \%$ & $17 \%$ & & \\
\hline BMI $\left(\mathrm{kg} / \mathrm{m}^{2}\right)$ & 23.2 & 25.7 & 40-45-year-old German females & German Federal Ministry of Health \\
\hline Present smoking (\%) & $11.8 \%$ & $28.4 \%$ & 40-49-year-old German females & $\begin{array}{l}\text { German Federal Ministry of Statistics } \\
2014\end{array}$ \\
\hline Netto household income $(€)$ & $€ 3700$ (mean) & $€ 3147$ (mean) & German population & $\begin{array}{l}\text { German Federal Ministry of Statistics } \\
2014\end{array}$ \\
\hline Education level & & & German population & German Federal Ministry of Statistics \\
\hline Very high & $47 \%$ & $29.5 \%$ & & 2015 \\
\hline High & $13 \%$ & $29.4 \%$ & & \\
\hline Low & $40 \%$ & $32.9 \%$ & & \\
\hline Risk drinking (> 10 g/day) & $1.5 \%$ & $22.1 \%$ & German female population & Robert Koch Institute 2014 \\
\hline
\end{tabular}


the age-adapted German reference group, the proportion of women who do not at all or rarely exercise was stated to be $47 \%$, a percentage of $25 \%$ performed $1-3 \mathrm{~h}$ physical training per week, $21 \%$ showed a training level of 3-5 h/week, and only $5 \%$ exercise more than $5 \mathrm{~h} /$ week.

\section{Smoking and alcohol consumption}

In the scope of our analysis, the rate of active smokers in our collective $(11.8 \%)$ was stated below the average of the German reference group of current smokers (28.4\% in 2014) as published by the German Federal Statistical Office.

In terms of alcohol consumption, only one participant indicated a risky alcohol consumption of more than $10 \mathrm{~g} /$ day. According to the Robert Koch Institute, the proportion of women with an alcohol consumption of more than $10 \mathrm{~g} /$ day was $23.12 \%$ in an age-adapted control group of German women 2014. However, as far as moderate alcohol consumption is concerned, $31 \%$ of our study participants stated to have a moderate alcohol consumption compared to a share of $52.3 \%$ of age-correlated German women [20].

\section{Socioeconomic background}

A total of 24 indicated to have more than the national average net income at their disposal, which is specified at $€ 3147$ or above per month. In addition, analysis showed no significant difference concerning the educational level in our study group compared to the German reference group, stating 47.0 vs. $29.5 \%$ of very high educational level, 13.0 vs. $29.4 \%$ of high educational level, and 40.0 vs. $32.9 \%$ of low educational level, respectively.

\section{Comparison of cancer diseased and non-cancer diseased mutation carriers}

As far as health consciousness and priorities in lifestyle intervention are concerned, some differences could be noticed between diseased and non-diseased mutation carriers. Cancer diseased mutation carriers seemed to have a lower BMI than non-diseased study participants, although this was not statistically significant $(p=0.079)$ (Tables 2 , 4). However, analyzing the motivation for physical activity, women without cancer in medical history revealed significantly higher levels of physical training per week compared to women that had already received a diagnosis of breast cancer (Tables 2, 4).

In the scope of physical activity, an additional analysis was then made examining the activity pattern in childhood and adolescence, in our study defined as the time period between the age of 10-19.

Accordingly, there is an indication for an important association between physical activity and cancer risk reduction by being more active than average during childhood and adolescence ( $p=0.019 *)$ (Tables 2, 4, Fig. 1).

Overall data illustrated a higher cancer prevalence in the study collective of present and former smokers $(p<0.001)$ compared to non-smokers. An association was not demonstrably attributable to the period of smoking time or age at smoking initiation (Tables 2, 3, 4).

In the context of analyzing the correlation of alcohol consumption and increase of cancer risk, this did not show statistical significance (Tables 2, 3, 4, Fig. 2).
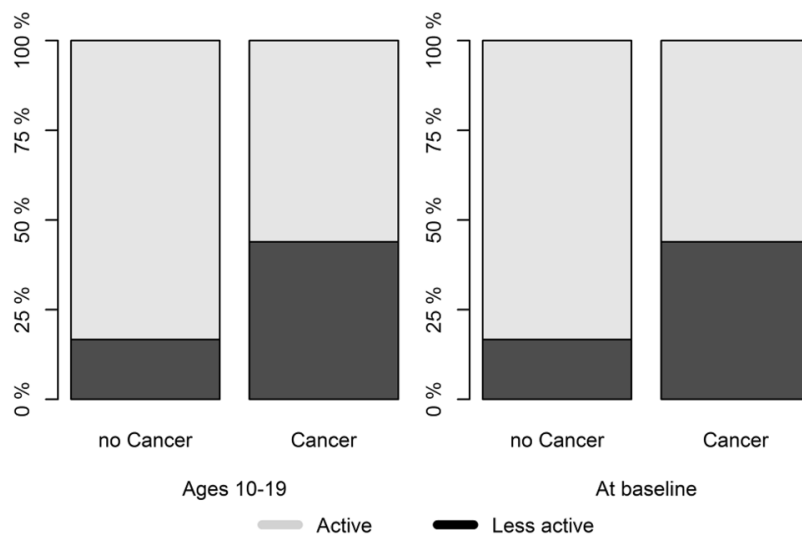

Fig. 1 Age-related physical activity of women diagnosed with cancer compared to women without cancer
Table 2 Comparison of diseased and non-diseased carriers at baseline

\begin{tabular}{llll}
\hline Parameter & $\begin{array}{l}\text { Diseased mutation carri- } \\
\text { ers } n=46\end{array}$ & $\begin{array}{l}\text { Non-diseased carriers } \\
n=22\end{array}$ & $p$ value \\
\hline Physical activity (h/week) at baseline & 8 & 15 & $\mathbf{0 . 0 4 6}$ \\
BMI $\left(\mathrm{kg} / \mathrm{m}^{2}\right)$ & 24 (mean) & 28 (mean) & 0.079 \\
BMI at study time $\left(\mathrm{kg} / \mathrm{m}^{2}\right)$ & $22.5 \pm 5$ (mean) & $25 \pm-8$ (mean) & 0.079 \\
BMI at age of 20 & $20.5 \pm 3$ & $22.5 \pm 5$ & 0.273 \\
Ever smoked & $n=35$ & $n=10$ & $\mathbf{0 . 0 2 3}$ \\
Income $(€)$ & 4157 (mean) & 3296 (mean) & 0.189 \\
\hline
\end{tabular}

Bold value indicates statistical significant 
Furthermore, we examined the socioeconomic background considering discrepancies in the collective of our

Table 3 Smoking behavior and alcohol consumption

\begin{tabular}{llll}
\hline & $\begin{array}{l}\text { Diseased muta- } \\
\text { tion carriers } \\
(n=46)\end{array}$ & $\begin{array}{l}\text { Non-diseased } \\
\text { mutation carriers } \\
(n=22)\end{array}$ & $p$ value \\
\hline $\begin{array}{l}\text { Present smoker } \\
\text { Former smoker }\end{array}$ & 6 & 2 & 0.535 \\
$\begin{array}{l}\text { Non-smoker } \\
\text { Age of smoking }\end{array}$ & 12 & 9 & 1.000 \\
$\quad$ begin & $16 \pm 4$ & 12 & - \\
$\begin{array}{l}\text { Number of years } \\
\text { smoked }\end{array}$ & $12.5 \pm 6$ & $8 \pm 9$ & 0.666 \\
$\begin{array}{l}\text { Alcohol consumption } \\
\text { Ever consumed } \\
\quad \text { alcohol }\end{array}$ & 45 & & 0.527 \\
$\begin{array}{l}\text { Consumption of } \\
\text { more than } 1 \mathrm{cl} \\
\text { glass/day at the }\end{array}$ & 8 & 22 & 1.000 \\
$\begin{array}{l}\text { age of 20 } \\
\text { Age at which } \\
\text { regular alcohol } \\
\text { consumption } \\
\text { began }\end{array}$ & 18.1 & 6 & 0.356 \\
\hline
\end{tabular}

Correlation is significant at the 0.05 level (twotailed) study participants. According to our data, diseased mutation carriers seemed to have lower households' incomes, but higher own incomes compared to healthy mutation carriers. Emphasizing the educational level, no difference could be demonstrated.

\section{Discussion}

Numerous studies demonstrate a noticeably increasing disease rate among BRCA mutation carriers. According to an Icelandic publication, a cumulative incidence of breast cancer (before the age of 70) between 1920 and 2000 among BRCA2 mutation carriers showed a fourfold increase (18.6 vs. 71.9\%) [15]. A similar escalation of risk occurred in the general population, showing that the cumulative incidence for sporadic breast cancer has increased from 1.8 to $7.5 \%$ over the last 80 years [15]. Overall, a clear increase of penetrance within the context of a BRCA mutation can be noted, whereby, besides genetic variants, this might be contingent on a change in reproductive behavior, as well as lifestyle factors.

However, possible modifying lifestyle risk factors have mainly been examined in retrospective case-control studies in mutation carriers, whereby the results were mostly distorted by bias in the clinical setting as well as survival.
Table 4 Association between breast cancer and lifestyle

\begin{tabular}{|c|c|c|c|c|}
\hline & Breast cancer & $n$ & Mean (SD) & $p$ value \\
\hline \multirow[t]{2}{*}{ Number of years smoked } & No & 11 & $12.4(8.8)$ & 0.808 \\
\hline & Yes & 33 & $12.9(6.0)$ & \\
\hline \multirow[t]{2}{*}{ Started smoking prior to breast cancer } & No & 0 & & $<0.001$ \\
\hline & Yes & 30 & & \\
\hline \multirow[t]{2}{*}{ More than a $1 \mathrm{cl}$ glass of alcohol per day at 20} & No & 7 & & 0.222 \\
\hline & Yes & 7 & & \\
\hline \multirow[t]{2}{*}{ BMI at age of 20} & No & 24 & $22.5(3.9)$ & 0.419 \\
\hline & Yes & 44 & $21.7(3.5)$ & \\
\hline \multirow[t]{2}{*}{ More active than average before 20} & No & 17 & & 0.016 \\
\hline & Yes & 21 & & \\
\hline \multirow[t]{2}{*}{ HRT ever taken } & No & 4 & & 0.185 \\
\hline & Yes & 3 & & \\
\hline \multirow[t]{2}{*}{ Use of hormonal contraception-years } & No & 22 & & 0.394 \\
\hline & Yes & 42 & & \\
\hline \multirow[t]{2}{*}{ Age at menarche } & No & 23 & $12.9(1.0)$ & 0.095 \\
\hline & Yes & 43 & $13.4(1.5)$ & \\
\hline \multirow[t]{2}{*}{ ABITUR achieved } & No & 13 & & 0.613 \\
\hline & Yes & 20 & & \\
\hline \multirow[t]{2}{*}{ Academic (i.e., university level) } & No & 13 & & 0.451 \\
\hline & Yes & 19 & & \\
\hline
\end{tabular}

Bold value indicates statistical significant

ABITUR qualification at the end of high or secondary school (ca. 18 years of age), HRT hormone replacement therapy 
Fig. 2 Influence of household income (left) and own net income (right) on cancer prevalence

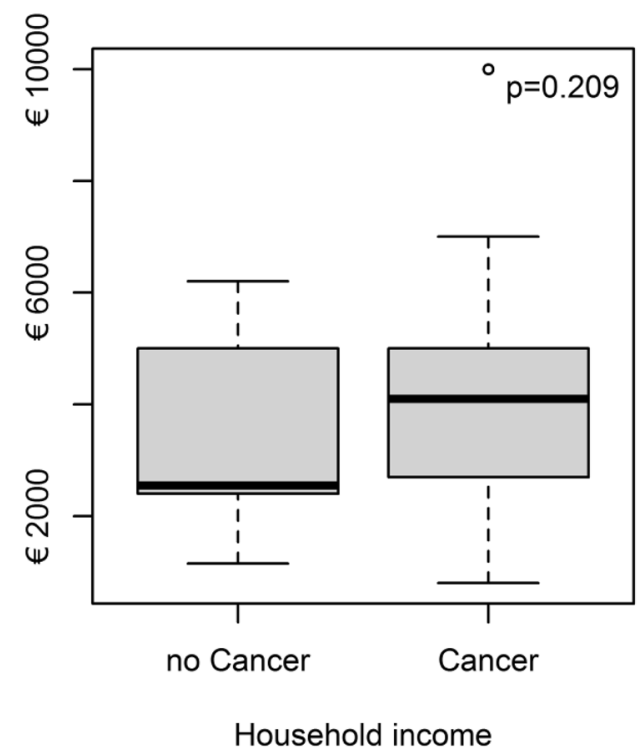

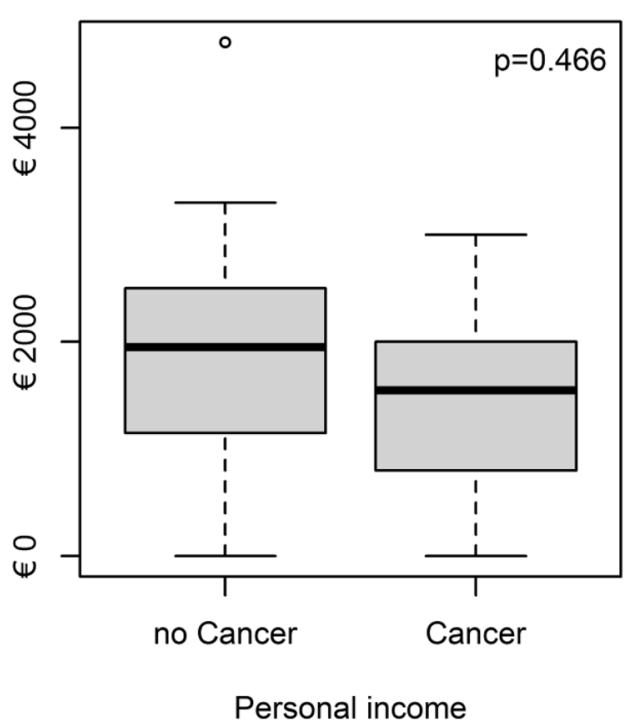

A validation of these findings through independent prospective studies has rarely taken place so far. The previous analyses yielded evidence of a presumable increase in risk through overweight, smoking, as well as lack of physical activity in the high-risk group of BRCA mutation carriers $[9,21]$. Nonetheless, the limitations of these studies are small sample sizes, meaning that large prospective studies are necessary to validate these assumptions in the group of genetically predisposed women. The LIBRE-1 study aimed to illustrate, in terms of a prospective, randomized feasibility study $(n=68)$, that a lifestyle intervention in the high-risk group of BRCA mutation carriers is possible. If this can be undertaken successfully, a validation of the modified lifestyle factors can be carried out with a large collective in the scope of LIBRE-2 with the aim of developing programs to reduce cancer incidence in BRCA mutation carriers.

Along with surveillance for BRCA mutation carriers, there is still a need for evidence-based recommendations regarding lifestyle choices besides the rather radical options of prophylactic surgery. Particularly, among women who are not opting to undergo prophylactic surgery or who are delaying surgery, we are in need of these alternative options to improve the physicians' counseling [9].

\section{Smoking}

According to current data, smoking can be defined as a risk factor for the development of sporadic breast cancer [22]. Smoking during adolescence up until the first pregnancy is considered particularly risky due to the increased susceptibility to chemical carcinogens of the, at this point, still incompletely differentiated mammary gland [23, 24].

To what extent smoking can also have an effect as a modulating factor in the scope of a strong genetic predisposition such as a BRCA mutation has been examined in few studies this far, and the existing data are inconsistent.

In 2009, Ginsburg et al. reported that there was no increase in the risk of breast cancer associated with current smoking in BRCA1 or BRCA2 carriers, based on their study of 2536 cases of breast cancer among women with a BRCA mutation [25]. Nevertheless, they noted that there seemed to be a possibility of an increased risk of breast cancer among BRCA1 carriers associated with past smoking [25]. Concluding it can be assumed that there are different effects of carcinogens in BRCA mutation carriers, depending on the timing of exposure [25].

Within our collective, $45 \%$ of the subjects stated that they had smoked in the past, at the beginning of the study $11.6 \%$ still smoked. Analyzing the data with respect to a smoking related increase in cancer prevalence, a statistically significant higher occurrence of cancer diseases could be determined in the collective of the BRCA positive smokers. However, an association between the intensity as well as the accumulated time period of nicotine consumption could not be found. This means that even in this small cohort, alone the habit of smoking can be considered as a significant risk factor for the high-risk collective of BRCA mutation carriers in developing carcinoma. Nonetheless, additional studies with a larger number of cases and an adjustment for other factors are necessary and already planned in the framework of the LIBRE 2 study.

In the scope of our analysis, we also noticed that a majority of the mutation carriers in our study collective already seem to display health-oriented efforts prior to the start of the study. They had already attempted to stop smoking by the time of the start of the study. The rate of active smokers in our collective (11.8\%) was below the average of the 
German reference groups of current smokers $(28.4 \%$ in 2014) as published by the German Federal Statistical Office.

\section{Body weight}

In the scope of large cohort studies, obesity as well as weight gain during adulthood are consistently associated with an increased risk, particularly for postmenopausal, sporadic breast cancer [26]. Weight gain of more than $20 \mathrm{~kg}$ during adulthood doubles the risk of developing sporadic breast cancer [26]. In the framework of the Women's Health Study, which examined more than 34,000 women, a $25-40 \%$ risk reduction could be demonstrated for postmenopausal breast cancer through a weight reduction of at least 5\% [26-29]. In the context of a meta-analysis, Suzuki et al. compared the highest versus the reference categories of relative body weight, showing that the risk for hormone receptor positive tumors was $20 \%$ lower among premenopausal and $82 \%$ higher among postmenopausal women [30]. To explain these contrary effects of BMI and obesity on breast cancer risk by menopausal status, for the most part, researchers have assumed that risk is moderated through an alteration in the source and levels of endogenous sex hormones, specifically estrogen as well as progesterone [9, 31]. Despite this, epidemiological evidence indicated an increased risk of triple-negative breast cancer with obesity, especially for premenopausal breast cancer [32].

Referring to $B R C A$ mutation carriers, there are also data, which provide important evidence that the maintenance of a BMI within the recommended limits may even reduce the incidence of high-risk BRCA-associated breast cancer [9].

Kotsopoulos et al. conducted a large study including 2146 BRCA mutation carriers as matched pairs [BRCA1 ( $n=797$ pairs), BRCA2 ( $n=276$ pairs)] [33]. Within this trial, a loss of at least $10 \mathrm{lb}(4.5 \mathrm{~kg})$ in the period between 18 and 30 years was associated with a decreased risk of breast cancer between the age of 30 and 49, whereas weight gain during the same interval did not influence the overall risk [33]. Nevertheless, large prospective studies are needed to evaluate the influence of body weight and weight gain or loss in BRCA mutation carriers.

Now focusing on our collective, the participants showed an average BMI of $23.2 \mathrm{~kg} / \mathrm{m}^{2}$ at the start of the study. In this regard, the mean BMI in our study was well below the average of the female German reference group according to the latest release of the German Federal Ministry of Health (2013).

According to subgroup analysis women already diagnosed with breast cancer tended to show a lower BMI than women without diagnosis of cancer. This could possibly mean that women who suffer from cancer may try to reduce possible risk factors yielding in a reduction of body weight (Table 2). Conversely, other hypotheses contend a possible risk reduction for premenopausal breast cancer by overweight, accentuating the mostly premenopausal age at the time of cancer diagnosis when carrying a BRCA mutation. To clarify this question, we will need more data, which we expect within the framework of LIBRE 2.

\section{Physical activity}

Numerous studies proved a risk-reducing effect of physical activity, particularly during adolescence, on the development of pre- and postmenopausal breast cancer, a risk reduction that was even present in patients with a familial predisposition, although less pronounced [34]. Potential protective effects are suspected in the reduction of the endogenous concentration of sex hormones, insulin resistance, as well as chronic inflammatory processes [34]. In addition, actual studies suggested that factors, which increase the physiologic expression of the normal copy of the BRCA1 or BRCA2 gene and thereby normalize protein levels, contribute to stem cell homeostasis, and/or affect hormone levels, and, therefore, might mitigate the effects of an inherited BRCA mutation [9]. In an in vivo study (Wang et al. 2009, an increase in BRCA1 mRNA expression could be demonstrated with increasing levels of physical activity [9]. Within this study, Wang et al. mentioned that physical activity, particularly during childhood, may be linked to a reduced risk of developing breast cancer in BRCA1 mutation carriers [9].

In our study group, $55.1 \%$ of the participants indicated that they had partaken in physical activity over average during their adolescence (ages 10-19) resulting in statistically significant fewer cancer diseases in a grouped analysis (Table 2, 4, Fig. 1). This finding commensurates Wang's hypothesis in 2009.

Moreover, analysis of the physical activity level of women who had already had a diagnosis of breast cancer revealed a significantly lower performance per week compared to women without breast cancer (Table 2, Fig. 1).

As described previously, our collective had already depicted impressive physical activity at the start of the study, $51 \%$ of the subjects indicated that they exercised one to $3 \mathrm{~h} /$ week, $21 \%$ even indicated a weekly activity level of $3-5 \mathrm{~h}$.

Comparing this activity level to the average German activity level, clear differences were to be noticed, where the proportion of women who were not doing any/rare sports was stated to be $47 \%$. In this average German collective, a percentage of $25 \%$ performed $1-3 \mathrm{~h}$ physical training per week, $21 \%$ showed a training level of 3-5 h/week, and only $5 \%$ exercised training of more than $5 \mathrm{~h} /$ week (Table 1 ).

\section{Alcohol consumption}

As already pointed out, risky and moderate alcohol consumption of our LIBRE-1 collective was far below the 
average of German women in 2014. In our analysis, we could not detect an alcohol-related change in breast cancer prevalence, which may be due to the small number of cases or the uniform levels of alcohol consumption in our population.

\section{Socioeconomic background}

In our analysis, there were no statistically significant findings regarding the influence of socioeconomic environment to cancer prevalence in BRCA mutation carriers. This will be further analyzed in the larger LIBRE-2 cohort [18].

In summary, the majority of the mutation carriers in our study seemed to be a rather health-oriented collective already before the start of the study. Lifestyle risk factors such as smoking and overweight were less prevalent than in peers. Physical activity was favored.

It seems that within our lifestyle intervention study, the mutation carriers at high risk for developing cancer disease might already have taken action to reduce cancer risks by a comparatively high level of health awareness. This might represent a selection bias of those, who consent to study participation. Digianni et al. [35] evaluated complementary and alternative medicine (including vitamins, special diets, herbal remedies, exercise, meditation/yoga, and acupuncture) by high-risk women before and 1 year following the confirmation of their BRCA1/2 genetic test results [9]. Women who are tested positive for a high-risk BRCA mutation frequently adopt new complementary and alternative medicine (CAM) use in the first year after learning about their genetic status considering these as part of their cancer preventive regimen [35].

This study likewise illustrates that after women receive a positive test result, they will modify their health practices and initiate supportive drugs for cancer prevention [9].

In particular, especially women diagnosed with cancer disease before, but also women who are aware of their high genetic cancer risks, show an outstanding interest in their health, resulting in a participation in physical training as well as awareness of nutrition and, therefore, body weight. This might also be their major motivation to participate in our lifestyle intervention trial (LIBRE-1). In this context, we must wait to see to what extent a significant improvement of the risk profile can be achieved through a lifestyle intervention conducted in our study, compared to an equally health-oriented control group. It can be assumed that so far two key events in BRCA mutation carriers sensitize them to lifestyle changes. The first is after they have been informed of the mutation and the second when they have been diagnosed with cancer. This information should be implemented in prevention programs for BRCA mutation carriers.

Despite careful attention to the methodology, there were a number of limitations that should be considered. This underlying analysis is a retrospective evaluation of clinical baseline characteristics of $B R C A$ mutation carriers that were enrolled in the LIBRE-1 study. On one hand, the selected sample (only women who decided to participate in the lifestyle intervention program were included) results in a group of overall high degree of motivation and less overall variability of motivation. On the other hand, the sample size is very low; a more representative sample size is planned in the LIBRE-2 study. Therefore, our current analysis must be considered as hypothesis generating emphasizing the limitations of the study.

Author contributions SG: data collection/analysis and manuscript writing. MY-D: data collection/analysis and manuscript editing. RD: data collection and manuscript writing/editing. JL: manuscript editing. MU: manuscript editing. CE: data collection and manuscript editing. KP: manuscript editing. MB: manuscript editing. MS: project development and manuscript editing. TS: manuscript editing. BW: manuscript editing. KR: manuscript editing. ND: manuscript editing. RS: manuscript editing. SCB: project development and manuscript editing. $\mathrm{MH}$ : project development and manuscript editing. MK: project development, funding acquisition, project manager, and manuscript editing.

\section{Compliance with ethical standards}

Funding This study was funded by the German Cancer Aid (Deutsche Krebshilfe), Project number: 110013.

Conflict of interest There is no conflict of interest.

Ethical approval All procedures performed in the LIBRE Study involving human participants were in accordance with the ethical standards of the institutional research committee and with the 1964 Helsinki declaration and its later amendments or comparable ethical standards. This chapter does not contain any studies with animals performed by any of the authors.

Informed consent Informed consent was obtained from all individual participants included in the study.

\section{References}

1. Ferlay J, Soerjomataram I, Dikshit R, Eser S, Mathers C, Rebelo $M$ et al (2015) Cancer incidence and mortality worldwide: sources, methods and major patterns in GLOBOCAN 2012. Int J Cancer 136(5):E359-E386

2. Ferrini K, Ghelfi F, Mannucci R, Titta L (2015) Lifestyle, nutrition and breast cancer: facts and presumptions for consideration. Ecancermedicalscience 9:557

3. Thomssen C, Wand D (2012) Hereditärer Brustkrebs. Der Onkologe 3(18):216-223

4. Mavaddat N, Peock S, Frost D, Ellis S, Platte R, Fineberg E et al (2013) Cancer risks for BRCA1 and BRCA2 mutation carriers: results from prospective analysis of EMBRACE. J Natl Cancer Inst 105(11):812-822

5. Vencken PM, Reitsma W, Kriege M, Mourits MJ, de Bock GH, de Hullu JA et al (2013) Outcome of BRCA1-compared with BRCA2-associated ovarian cancer: a nationwide study in the Netherlands. Ann Oncol 24(8):2036-2042 
6. Antoniou AC, Beesley J, McGuffog L, Sinilnikova OM, Healey S, Neuhausen SL et al (2010) Common breast cancer susceptibility alleles and the risk of breast cancer for BRCA1 and BRCA2 mutation carriers: implications for risk prediction. Cancer Res 70(23):9742-9754

7. King MC, Marks JH, Mandell JB, New York Breast Cancer Study $\mathrm{G}$ (2003) Breast and ovarian cancer risks due to inherited mutations in BRCA1 and BRCA2. Science 302(5645):643-646

8. Bissonauth V, Shatenstein B, Fafard E, Maugard C, Robidoux A, Narod S et al (2009) Weight history, smoking, physical activity and breast cancer risk among French-Canadian women non-carriers of more frequent BRCA1/2 mutations. J Cancer Epidemiol 2009:748367

9. Pettapiece-Phillips R, Narod SA, Kotsopoulos J (2015) The role of body size and physical activity on the risk of breast cancer in BRCA mutation carriers. Cancer Causes Control 26(3):333-344

10. Renehan AG, Tyson M, Egger M, Heller RF, Zwahlen M (2008) Body-mass index and incidence of cancer: a systematic review and meta-analysis of prospective observational studies. Lancet 371(9612):569-578

11. Chen WY, Rosner B, Hankinson SE, Colditz GA, Willett WC (2011) Moderate alcohol consumption during adult life, drinking patterns, and breast cancer risk. JAMA 306(17):1884-1890

12. Howell A, Anderson AS, Clarke RB, Duffy SW, Evans DG, Garcia-Closas $\mathrm{M}$ et al (2014) Risk determination and prevention of breast cancer. Breast Cancer Res 16(5):446

13. Antoniou A, Pharoah PD, Narod S, Risch HA, Eyfjord JE, Hopper JL et al (2003) Average risks of breast and ovarian cancer associated with BRCA1 or BRCA2 mutations detected in case Series unselected for family history: a combined analysis of 22 studies. Am J Hum Genet 72(5):1117-1130

14. Rebbeck TR, Mitra N, Domchek SM, Wan F, Friebel TM, Tran TV et al (2011) Modification of BRCA1-associated breast and ovarian cancer risk by BRCA1-interacting genes. Cancer Res 71(17):5792-5805

15. Tryggvadottir L, Sigvaldason H, Olafsdottir GH, Jonasson JG, Jonsson T, Tulinius H et al (2006) Population-based study of changing breast cancer risk in Icelandic BRCA2 mutation carriers, 1920-2000. J Natl Cancer Inst 98(2):116-122

16. Rebbeck TR, Wang Y, Kantoff PW, Krithivas K, Neuhausen SL, Godwin AK et al (2001) Modification of BRCA1- and BRCA2associated breast cancer risk by AIB 1 genotype and reproductive history. Cancer Res 61(14):5420-5424

17. Kiechle M, Engel C, Berling A, Hebestreit K, Bischoff S, Dukatz $R$ et al (2016) Lifestyle intervention in BRCA1/2 mutation carriers: study protocol for a prospective, randomized, controlled clinical feasibility trial (LIBRE-1 study). Pilot Feasibility Stud 2:74

18. Kiechle M, Engel C, Berling A, Hebestreit K, Bischoff SC, Dukatz $\mathrm{R}$ et al (2016) Effects of lifestyle intervention in BRCA1/2 mutation carriers on nutrition, BMI, and physical fitness (LIBRE study): study protocol for a randomized controlled trial. Trials 17:368

19. Schag CC, Heinrich RL, Ganz PA (1984) Karnofsky performance status revisited: reliability, validity, and guidelines. J Clin Oncol 2(3):187-193
20. Lange C, Manz K, Rommel A, Schienkiewitz A, Mensink GBM (2016) J Health Monit 1(1). https://www.rki.de/DE/Content/ Gesundheitsmonitoring/Gesundheitsberichterstattung/GBEDownloadsJ/JoHM_2016_01_alkohol.pdf?_blob=publicationFile. Accessed 29 Aug 2017

21. Friebel TM, Domchek SM, Rebbeck TR (2014) Modifiers of cancer risk in BRCA1 and BRCA2 mutation carriers: systematic review and meta-analysis. J Natl Cancer Inst. 106(6):dju091

22. Catsburg C, Kirsh VA, Soskolne CL, Kreiger N, Rohan TE (2014) Active cigarette smoking and the risk of breast cancer: a cohort study. Cancer Epidemiol 38(4):376-381

23. Reynolds P (2013) Smoking and breast cancer. J Mammary Gland Biol Neoplas 18(1):15-23

24. Glantz SA, Johnson KC (2014) The surgeon general report on smoking and health 50 years later: breast cancer and the cost of increasing caution. Cancer Epidemiol Biomark Prev 23(1):37-46

25. Ginsburg O, Ghadirian P, Lubinski J, Cybulski C, Lynch H, Neuhausen $S$ et al (2009) Smoking and the risk of breast cancer in BRCA1 and BRCA2 carriers: an update. Breast Cancer Res Treat 114(1):127-135

26. Vrieling A, Buck K, Kaaks R, Chang-Claude J (2010) Adult weight gain in relation to breast cancer risk by estrogen and progesterone receptor status: a meta-analysis. Breast Cancer Res Treat 123(3):641-649

27. Eliassen AH, Colditz GA, Rosner B, Willett WC, Hankinson SE (2006) Adult weight change and risk of postmenopausal breast cancer. JAMA 296(2):193-201

28. van den Brandt PA, Spiegelman D, Yaun SS, Adami HO, Beeson L, Folsom AR et al (2000) Pooled analysis of prospective cohort studies on height, weight, and breast cancer risk. Am J Epidemiol 152(6):514-527

29. Scoccianti C, Lauby-Secretan B, Bello PY, Chajes V, Romieu I (2014) Female breast cancer and alcohol consumption: a review of the literature. Am J Prev Med 46(3 Suppl 1):S16-S25

30. Suzuki R, Orsini N, Saji S, Key TJ, Wolk A (2009) Body weight and incidence of breast cancer defined by estrogen and progesterone receptor status - a meta-analysis. Int J Cancer 124(3):698-712

31. Potischman N, Swanson CA, Siiteri P, Hoover RN (1996) Reversal of relation between body mass and endogenous estrogen concentrations with menopausal status. J Natl Cancer Inst 88(11):756-758

32. Pierobon M, Frankenfeld CL (2013) Obesity as a risk factor for triple-negative breast cancers: a systematic review and meta-analysis. Breast Cancer Res Treat 137(1):307-314

33. Kotsopoulos J, Olopado OI, Ghadirian P, Lubinski J, Lynch HT, Isaacs $C$ et al (2005) Changes in body weight and the risk of breast cancer in BRCA1 and BRCA2 mutation carriers. Breast Cancer Res 7(5):R833-R843

34. Lynch BM, Neilson HK, Friedenreich CM (2011) Physical activity and breast cancer prevention. Recent Results Cancer Res 186:13-42

35. Digianni LM, Rue M, Emmons K, Garber JE (2006) Complementary medicine use before and 1 year following genetic testing for BRCA1/2 mutations. Cancer Epidemiol Biomark Prev 15(1):70-75 